\title{
Crohn's-like disease in a patient with common variable immunodeficiency treated with azathioprine and adalimumab
}

\author{
Juan María Vázquez-Morón ${ }^{1}$, Héctor Pallarés-Manrique ${ }^{1}$, Ignacio Javier Martín-Suárez², \\ Beatriz Benítez-Rodríguez ${ }^{1}$ and Manuel Ramos-Lora ${ }^{1}$ \\ Departments of ${ }^{l}$ Gastroenterology and ${ }^{2}$ Internal Medicine. Hospital Juan Ramón Jiménez. Huelva, Spain
}

\begin{abstract}
Common variable immunodeficiency (CVID) is the most frequent primary antibody deficiency. It is characterized by recurrent bacterial infections, and occurrence of autoimmune and neoplastic diseases is also frequent; there is also a high prevalence of gastrointestinal diseases. There are reports of inflammatory bowel disease in this entity, but incidence is low (2-4\%). We present the case of a patient with common variable immunodeficiency suffering a chronic diarrhoea episode and who was diagnosed with ileocaecal Crohn s-like disease after performing intestinal transit, CT abdomen and colonoscopy with biopsy. It was first treated with prednisone but on showing cortisone dependency, treatment with azathioprine and adalimumab was started, with good results.
\end{abstract}

Key words: Crohn's-like disease. Common variable immunodeficiency. Adalimumab. Azathioprine.

\section{INTRODUCTION}

Common variable immunodeficiency (CVID), also called acquired hypogammaglobulinemia, is the most common primary antibody deficiency. It can occur at any age but is

Vázquez-Morón JM, Pallarés-Manrique H, Martín-Suárez IJ, Benítez-Rodríguez B, Ramos-Lora M. Crohn's-like disease in a patient with common variable immunodeficiency treated with azathioprine and adalimumab. Rev Esp Enferm Dig 2013;105: 299-302.

Received: $25-10-2012$

Accepted: $19-11-2012$

Correspondence: Juan María Vázquez-Morón. Department of Gastroenterology. Hospital Juan Ramón Jiménez. Ronda Norte, s/n. 21005 Huelva, Spain e-mail: juanma_cartaya@hotmail.com most common among people between twenty and fifty years old, with equal incidence in both sexes. It is diagnosed after finding decreases, below two standard deviations, of at least two of the three major classes of immunoglobulins ( $\operatorname{IgA}$, $\operatorname{IgG}, \operatorname{IgM}$ ), a deterioration of the production of antibodies in response to infection and/or vaccines because of failure in the differentiation of B lymphocytes from plasma cells, and a normal or slightly decreased number of T and B lymphocytes circulating (1).There is a high prevalence of gastrointestinal diseases. Up to $60 \%$ of untreated patients may have diarrhoea. There are reports of inflammatory bowel disease associated with this entity; however, incidence is low $(2-4 \%)$. Crohn's-like disease is the appearance of an inflammatory bowel disease, which is compatible with Crohn's disease as a consequence of another underlying pathology. Autoimmune phenomena occur with some frequency in patients with immunodeficiencies. Specifically, in CVID it has been observed in a group of patients that activation of tumour necrosis factor alpha (TNF $\alpha$ ) persists, contributing to the onset of inflammatory bowel disease in these patients (2). We present a case of Crohn's-like disease in a patient with common variable immunodeficiency treated with adalimumab and azathioprine with good outcome.

It is an unusual case, and the first one published in our country that was treated with adalimumab and showed good response.

\section{CASE REPORT}

A 33 year-old male, smoker, with a history of allergy to aspirin and common variable immunodeficiency syndrome diagnosed at 6 years of age based on laboratory (IgA and IgM levels below normal) and clinical criteria (multiple infectious processes in childhood), currently in intravenous immunoglobulin replacement therapy at a 


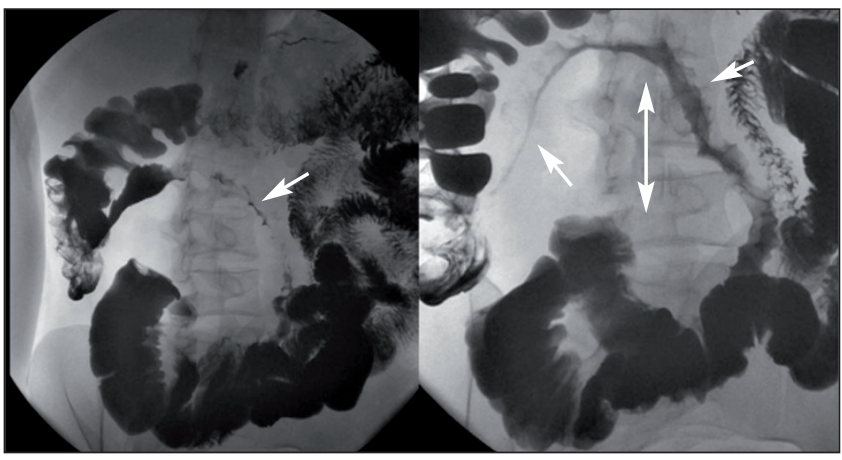

Fig. 1. Image of barium bowel transit which shows a large segment of terminal distal ileum with loss of distensibility and contractility, as well as fibrino-estenotic changes and the presence of deep ulcers compatible with Crohn's disease with ileal involvement.

dose of 12,000 IU every 21 days. The patient had lung bronchiectasis, sinonasal polyposis, gastroesophageal reflux, platelet adhesion deficiency, hip arthritis, phlebitis and cellulitis in lower members and was operated on for cryptorchidism in childhood.

The patient went to the emergency room because he was suffering from diarrhoea, without blood or pathological products. The diarrhoea was accompanied by abdominal pain in the right lower quadrant which had built up over 5 months and the patient complained he had lost about $15 \mathrm{~kg}$ of weight in this period. Physical examination showed the patient was afebrile and haemodynamically stable, rhonchi could be heard in both lung bases, and palpation showed abdominal pain in the right lower quadrant without signs of peritonitis.

The haemogram showed $\mathrm{Hb}$ of $11.5 \mathrm{~g} / \mathrm{dL}$, haematocrit $33 \%$, MCV $73 \mathrm{fl}, \mathrm{WBC} 25,800 / \mathrm{mm}^{3}$ (84\% segmented), platelet $516,000 / \mathrm{mm}^{3}$. In biochemistry the elevated acute phase reactants (CRP $5.6 \mathrm{mg} / \mathrm{dL}$, ESR $36 \mathrm{~mm} / \mathrm{h}$, alfa1glicoproten acid $216 \mathrm{mg} / \mathrm{dl}$ and faecal calprotectin $1,640 \mathrm{mg} / \mathrm{g}$ ) were remarkable; the other biochemical parameters showed normal levels, as did the thyroid hormone. The proteinogram reported a moderate inflammatory profile with IgM $1 \mathrm{mg} / \mathrm{dL}$ (56-352 mg/dL), $807 \mathrm{IgG} \mathrm{mg} / \mathrm{dL}$ (640 to 1,350 $\mathrm{mg} / \mathrm{dL}), \operatorname{IgA} 7 \mathrm{mg} / \mathrm{dL}(70-132 \mathrm{mg} / \mathrm{dL})$. The stool culture and serology for HBV, HCV and HIV was negative. The ANA showed 1/80 with antiDNA negative and negative IgG transglutaminase antibodies. The mantoux test was also negative. In the chest X-ray, the only findings were bronchiectasis in both lungs. Intestinal transit was performed (Fig. 1) and a CT of the abdomen (Fig. 2) where there were signs compatible with inflammatory bowel disease Crohn's-like disease at ileal and caecal levels. Colonoscopy with ileoscopy showed congestive mucosa with several shallow superficial ulcers with fibrinous surface (Fig. 3); biopsies were taken at both levels. The anatomical-pathological examination of the samples reported intestine mucosa with fibrin deposition, leukocyte accumulation and granulation tissue showing architectural distortion of the crypts with no plasma cells present (Fig. 4).
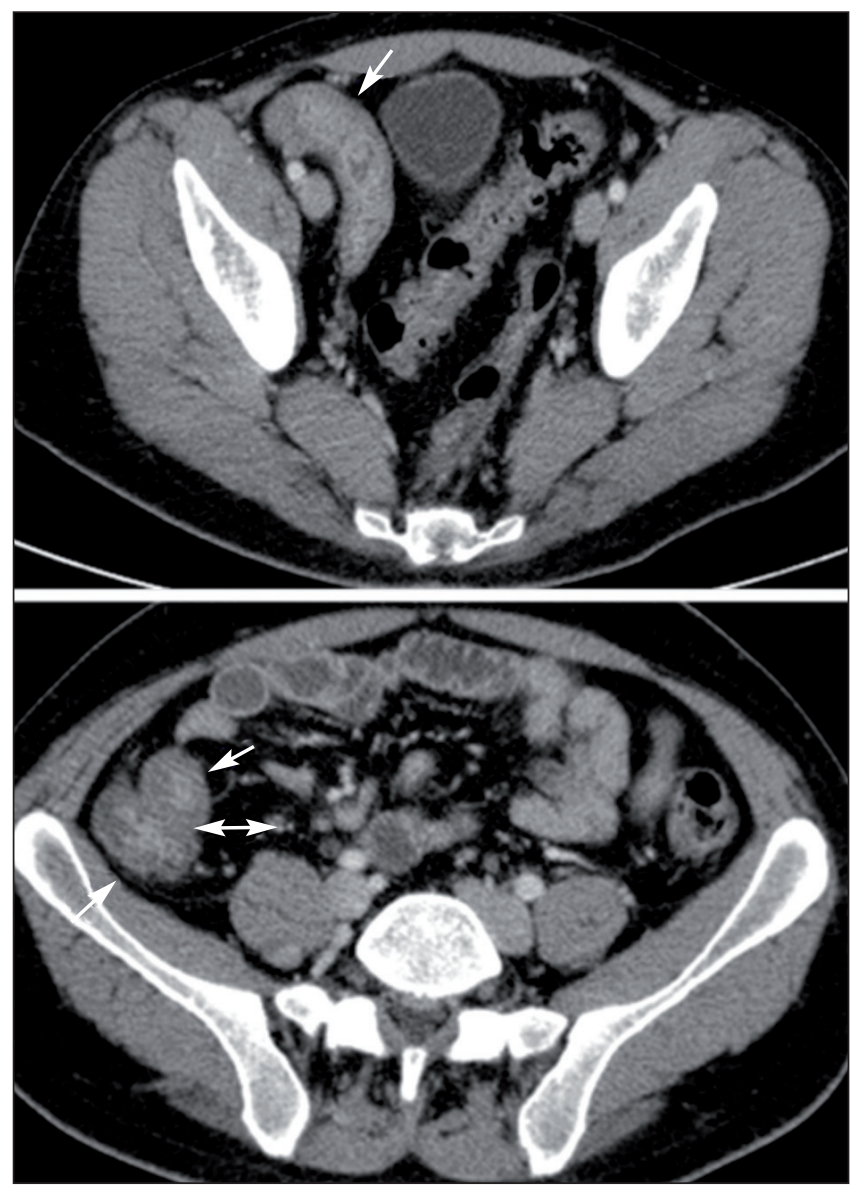

Fig. 2. Abdominal CT image is observed in the circumferential thickening of the terminal ileum and caecum region with faint glare of the adjacent fat and reactive lymph node.

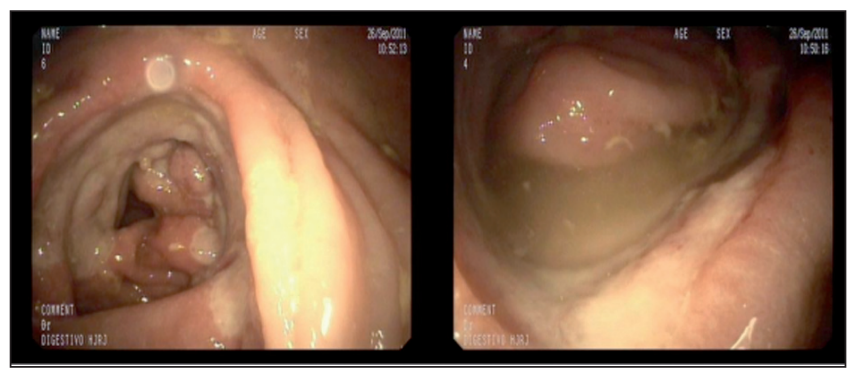

Fig. 3. Endoscopic image of extensive shallow fibrinoid ulcers at the cecum and ileocecal valve.

Mycobacterial culture was performed on sputum and ZiehlNielsen staining in intestinal tissue samples was negative. All these data led to the diagnosis of Crohn's-like disease in a patient with common variable immunodeficiency indicating treatment with prednisone at a dose of $1 \mathrm{mg} / \mathrm{kg}$ for 2 weeks and with gradual reduction of dosage. However, the patient showed corticosteroid dependency and the daily dose of $20 \mathrm{mg}$ of prednisone could not be lowered without clinical worsening appearing as recurrent abdominal pain 


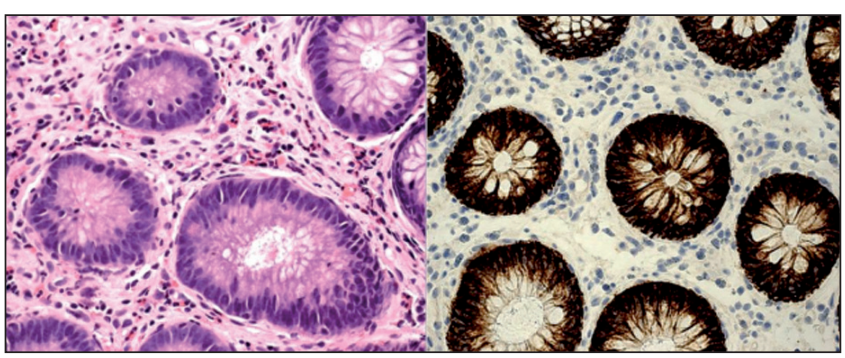

Fig. 4. Histological image of the large intestine mucosa with fibrino-leukocytic material and granulation tissue in which no plasma cells are observed.

and loose stools. It was therefore decided to start treatment with azathioprine and adalimumab with $80 \mathrm{mg}$ induction and $40 \mathrm{mg}$ every two weeks for compassionate use. After several weeks of treatment, the patient showed an improvement in clinical symptoms, reduction of acute phase reactants (CRP $0.4 \mathrm{mg} / \mathrm{dL}$ and ESR $21 \mathrm{~mm} / \mathrm{h}$ ) and decreased wall thickening at ileocecal level in the in control CT. No adverse effects were observed in this period.

\section{DISCUSSION}

Common variable immunodeficiency (CVID) is characterized by recurrent bacterial infections, particularly of the upper and lower respiratory tract, and is also associated to an increase in neoplastic and autoimmune diseases ${ }^{3}$. There is a high prevalence of gastrointestinal diseases. Up to $60 \%$ of untreated patients with CVID develop diarrhoea and $10 \%$ develop idiopathic malabsorption associated with weight loss, giardiasis and bacterial overgrowth in the small intestine being the most common cause of these manifestations. Inflammatory processes are also common and can appear as nodular lymphoid hyperplasia, aphthous stomatitis, autoimmune atrophic gastritis, pernicious anaemia, chronic enteritis, inflammatory bowel disease including chronic hepatitis. An increase in the frequency of gastrointestinal tumours such as lymphomas and adenocarcinomas (4) is detected.

Several studies have documented increased incidence of inflammatory bowel disease (IBD), Crohn's-like disease or ulcerative colitis, in CVI patients (4-6). In fact, the concomitant presence of IBD patients is higher than the incidence of IBD in the general population. In Spain, in a retrospective study estimated the prevalence of IBD in patients with CVID was around $3.2 \%$ (7). The most prevalent cause of this is not known, although it is suggested the existence of an immune dysregulation that can be expressed as an intestinal inflammatory process in these patients. Although a decrease in cytokines is usually apparent in CVID (principally IL-2, IL-10, TNF $\alpha$ ) produced by T cells, reduction of serum immunoglobulins and impaired antibody response (8).

The main clinical manifestations presented by patients with Crohn's-like disease in a CVID context are chronic diarrhoea, weight loss and malabsorption (9) as in our patient. Occasionally gastrointestinal symptoms may develop before the underlying immunodeficiency is discovered. Various clinical manifestations of CVID suggest that the pathogenesis of this disorder is complex, with autoimmune alterations appearing in multiple pathways. Identification of immunological parameters associated with this subgroup of patients may allow early diagnosis and help establish more specific and targeted treatment (10).

The findings that are seen in the colonoscopy are those of classical Crohn's disease showing congested mucosa accompanied by erosions and fibrinoid ulcers (11). Histological examination reveals a number of features that help confirm the diagnosis, such as the presence of prominent lymphoid aggregates, architectural distortion accompanied by cryptitis and non-caseating granulomas have even been described in around $20 \%$ of cases. One of the key features in Crohn'slike disease in CVID is the appearance of a marked decrease of plasma cells in approximately $67 \%$ of cases (12).

Treatment in these patients is not established as there have been a limited number of cases, all of which have received empirical treatment. In addition to intravenous immunoglobulin replacement therapy, steroids or immunosuppressive drugs have been used to control diarrhoea but their efficacy and tolerability are not well documented. Some cases of patients with diarrhoea and CVID who have been treated with oral budesonide, reducing the diarrhoea without presenting any side effects have been reported $(13,14)$. Cases have also been reported in patients with Crohn's-like disease in context of CVID like who have been treated with infliximab; in particular in two cases in which infliximab and azathioprine treatment was used disease activity remitted and no adverse effect was reported (15). We began treating our patient with azathioprine and adalimumab; this is the first case reported in our country.

\section{REFERENCES}

1. Park MA, Li JT, Hagan JB, Maddox DE, Abraham RS. Common variable immunodeficiency: a new look at an old disease. Lancet 2008 ; 372:489-502.

2. Aukrust P, Lien E, Kristoffersen AK, Müller F, Haug CJ, Espevik T, et al. Persistent activation of the tumor necrosis factor system in a subgroup of patients with common variable immunodeficiency. Possible immunologic and clinical consequences. Blood 1996;87:674-81.

3. Hermaszewski RA, Webster ADB. Primary hypogammaglobulinemia: A survey of clinical manifestations and complications. Q J Med 1993;86:31-42.

4. Khodadad A, Aghamohammadi A, Parvaneh N, Rezaei N, Mahjoob F, Bashashati M, et al. Gastrointestinal manifestations in patients with common variable immunodeficiency. Dig Dis Sci 2007;52:2977-83.

5. Cunningham-Rundles $\mathrm{C}$, Bodian C. Common variable immunodeficiency: Clinical and immunological features of 248 patients. Clin Immunol 1999;92:34-48.

6. Quinti I, Soresina A, Spadaro G, Martino S, Donnano S, Agostini C, et al. Long-term follow-up and outcome of a large cohort of patients with common variable immunodeficiency. J Clin Immunol 2007; 27:308-16.

7. Sarmiento E, Mora R, Rodríguez-Mahou M, Rodríguez-Molina J, Fernández-Cruz E, Carbone J. Autoinmune disease in primary antibody deficiencies. Allergol immunopathol 2005;33:69-73. 
8. Cunningham-Rundles C. Common variable immunodeficiency. Curr Allergy Asthma Rep 2001;1:421-9.

9. Bosworth BP, Sanders A, Maltz C. Common variable immunodeficiency masquerading as Crohn sileocolitis. Inflamm Bowel Dis 2006; 12:151-2.

10. Daniels JA, Lederman HM, Maitra A, Montgomery EA. Gastrointestinal tract pathology in patients with common variable immunodeficiency (CVID): A clinicopathologic study and review. Am J Surg Pathol 2007;31:1800-12.

11. Mudter J, Wirtz S, Weigmann B, Tiede I, Tubbe I, Kiesslich R, et al. Crohn's-like colitis in a patient with immunodeficiency associated with a defect in expression of inducible costimulator. Dig Dis Sci 2006; 51:711-7.
12. Daniels JA, Lederman HM, Maitra A, Montgomery EA. Gastrointestinal tract pathology in patients with common variable immunodeficiency (CVID): A clinicopathologic study and review. Am J Surg Pathol 2007;31:1800-12.

13. Elnachef N, McMorris M, Chey W. Successful treatment of common variable immunodeficiency disorder-associated diarrhea with budesonide: A case report. Am J Gastroenterol 2007;102:1322-5.

14. Córdoba Guevara H, Guarner Aguilar L. Diarrea asociada a inmunodeficiencia común variable tratada con budesonida. Rev Esp Enferm Dig 2009;101:215-9.

15. Nos P, Bastida G, Beltran B, Aguas M, Ponce J. Crohn's disease in common variable immunodeficiency: Treatment with antitumor necrosis factor alpha. Am J Gastroenterol 2006;101:2165-6. 